\title{
X-ray Spectrum Imaging at High Resolution in the STEM and STEM/SEM and SEM
}

\author{
James Sagar ${ }^{1}$, Dylan Wood ${ }^{2}$, Philippe Pinard ${ }^{1}$, Jane Howe ${ }^{3}$, James Holland ${ }^{1}$, Simon Burgess ${ }^{1}$ and Peter Statham ${ }^{1}$ \\ 1. Oxford Instruments Nanoanalysis, High Wycombe, United Kingdom. \\ 2. Oxford Instruments Inc., Concord, USA. \\ 3. Hitachi High-Technologies America Inc., Clarksburg, USA.
}

With the advent of large area silicon drift detectors X-ray microanalysis has moved from a technique based on point spectra to one focused more on mapping element distributions. With this change in focus comes a new drive to image using energy dispersive $x$-ray spectrometry (EDS) at increasing spatial resolution. The standard technique for high resolution EDS, e.g. for features of less than $20 \mathrm{~nm}$, is STEM at high voltage, typically $200 \mathrm{kV}$ or greater. Using aberration corrected STEM at high $\mathrm{kV}$, it has been demonstrated that atomic resolution EDS is achievable in a wide range of specimens [1,2]. It is also understood that at this resolution EDS is no longer a quantitative technique with the effects of electron channelling dominating the relative X-ray intensity between atoms [3]. The achieved spectrum images do however give excellent information on the spatial distribution of elements. If absolute quantitative understanding of elemental distribution is not required then developments in lower $\mathrm{kV}$ electron microscopy technology may provide a real alternative to high voltage, to achieve nanometre resolution EDS mapping.

For example, developments in FIB technology mean that lamellae of $<50 \mathrm{~nm}$ in thickness are now routinely produced. At this sample thickness, the beam spread through the specimen is much larger at $30 \mathrm{kV}$ than at $200 \mathrm{kV}$. However, Monte-Carlo simulations (figures 1a, 1c, 1e) shows that the region from which $>70 \%$ of X-rays are generated at $30 \mathrm{kV}$ is still less than $10 \mathrm{~nm}$ which is often adequate to achieve similar spatial resolution of Xray spectrum images. Simulations show that for some specimens it can actually be beneficial to use $30 \mathrm{kV}$ STEM because of the increase in X-ray ionization cross-sections. Our simulations for a pure Ni lamella show that a 2.3 times increase in the Ni Ka X-ray count rate can be expected for $30 \mathrm{kV}$ over $200 \mathrm{kV}$.

To investigate this we have tested a Ni superalloy 718 sample using different methodologies to achieve high spatial resolution spectrum images. This specimen was chosen as it contained a number of different phases that form nanometre scale precipitates. The elements within the specimen also have available X-ray lines for all elements below $3 \mathrm{keV}$ allowing us to compare the resolution of spectrum images at extremely low beam energy as well as the higher energies previously discussed. This is important as for a significant number of applications thinning specimens to electron transparency is not possible or practical.

The Ni superalloy specimen was prepared by electropolishing, to create a large electron transparent area that could be analysed in different tools to compare the spatial resolution of EDS spectrum images. All EDS measurements were performed using either single or dual $100 \mathrm{~mm}^{2}$ windowless silicon drift detectors. These detectors achieve very large collection solid angles and their windowless nature ensures efficient collection of low energy X-rays, either from light elements or from the L, M and N lines of heavy elements. In a TEM at $200 \mathrm{kV}$, two detectors were positioned $180^{\circ}$ to each other achieving a combined solid angle of 2 steradians. In the SEM, for measurements at $30 \mathrm{kV}$ and $3 \mathrm{kV}$, a single detector was used with a solid angle of 0.7 steradians.

Using these different instruments EDS spectrum images were collected. From these the phases detected have been compared, as well as the achieved spatial resolution. In figures 1d-f we show that it is possible to resolve $\sim 10 \mathrm{~nm}$ $\gamma$ ' precipitates from the matrix at $200 \mathrm{kV}, 30 \mathrm{kV}$ and $3 \mathrm{kV}$ using EDS. Comparing the EDS spectrum images with simulations allows us understand the practical limits to EDS spectrum image resolution, and explore the limit of phase detection of these techniques. By doing this we can understand how much of the work that is currently done 
at $200 \mathrm{kV}$ STEM could in fact now be successfully carried out either at $30 \mathrm{kV}$ on a thinned sample or even at $3 \mathrm{kV}$ on a bulk sample in an SEM.

References:

[1] A. J. D’Alfonso et al, Phys. Rev. B 81 (2010), p. 100101(R).

[2] P. Lu et al, Scientific Reports 4 (2014), p. 3945.

[3] Z. Chen et al, Ultramicroscopy 176 (2017), p. 52.

[4] N. W. M. Ritchie, Surface and Interface Analysis 37 (2005), p. 1006.
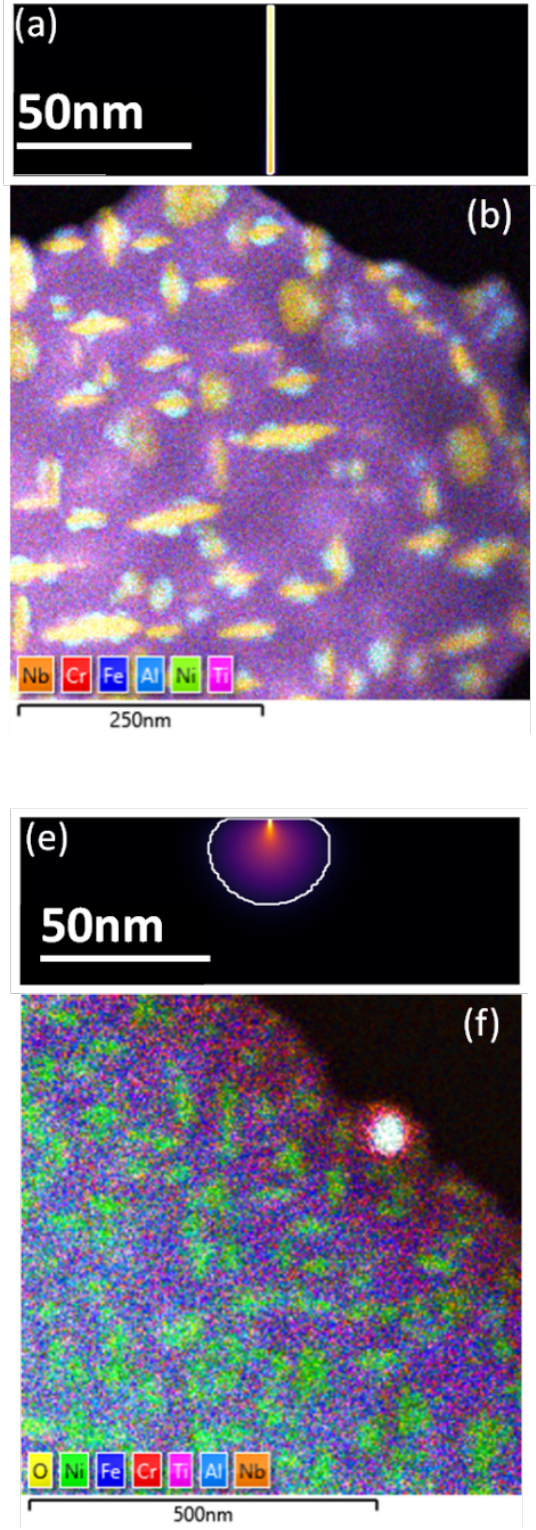
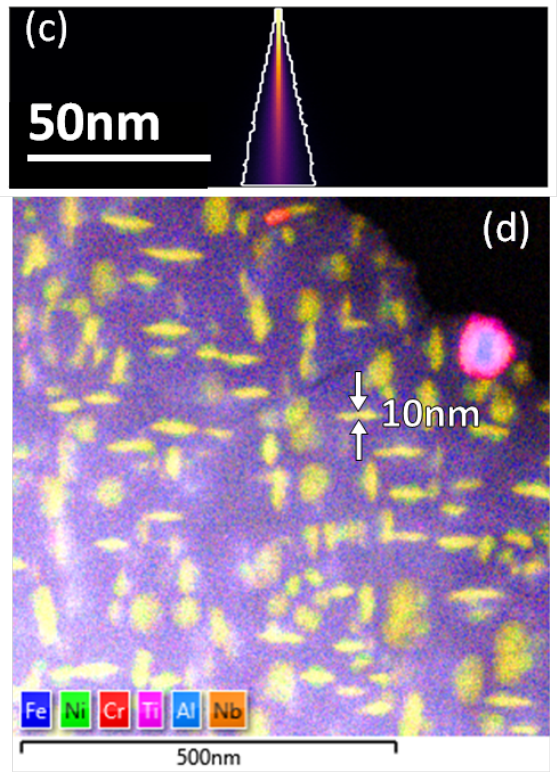

Figure 1. Monte Carlo simulations with NISTMonte [4] of Ni Ka X-ray generation volume for an electron probe of a) $200 \mathrm{kV} \mathrm{c}$ ) $30 \mathrm{kV}$ and $\mathrm{Ni} \mathrm{L} \alpha \mathrm{X}$-ray generation volume for an electron probe of e) $3 \mathrm{kV}$ in a $50-\mathrm{nm}$ thick pure Ni lamella. EDS spectrum images of $\mathrm{Ni}$ Superalloy 718 acquired at b) $200 \mathrm{kV}$ (Hitachi HF5000 probe corrected S/TEM) d) $30 \mathrm{kV}$ and f) $3 \mathrm{kV}$ (Hitachi SU9000 STEM/SEM) with 30 minute acquisition time. 